\title{
Characteristics of a deep crack occurred on intact molars without restoration
}

\author{
Soo-Jeong Hwang ${ }^{1}$, Min-Seock Seo ${ }^{2 *}$ \\ ${ }^{1}$ Department of Dental Hygiene, College of Medical Science, Konyang University, Daejeon, Korea \\ ${ }^{2}$ Department of Conservative Dentistry, Wonkwang University Daejeon Dental Hospital, Daejeon, Korea
}

The objective of this study was to investigate the characteristics of a deep crack in intact teeth with no restoration. The molars which were treated endodontically in the Department of Conservative Dentistry at Wonkwang University Daejeon Dental Hospital were screened and evaluated from January 2017 to June 2018. Clinical records of endodontically treated molars were reviewed and the molars diagnosed as cracked tooth were selected. From clinical records, only the cases where the crack line was confirmed to extend to the wall of access cavity were included in the study. Teeth with dental caries, restorations, or fractures at the time of diagnosis were excluded. General and pretreatment data of 85 cracked teeth were collected from the clinical records. The relatively deep cracks from intact molars occurred two times more in the maxillary molars than in the mandibular molars, and five times more in males than in females. Crack lines observed from access cavity wall were significantly different in maxillary and mandibular molars. The contralateral symmetrical molars of the cracked tooth had 'crack or fracture' or was 'extracted' at a higher rate than the occluding teeth. Deep cracks in intact molar had different characteristics from general cracks. In case someone has a cracked tooth, it may be important to check the condition of the contralateral tooth and consider the intervention.

Key Words: Cracked tooth syndrome, Endodontics, Molar

(c) This is an open-access article distributed under the terms of the Creative Commons Attribution Non-Commercial License (http://creativecommons.org/licenses/by-nc/4.0) which permits unrestricted noncommercial use, distribution, and reproduction in any medium, provided the original work is properly cited.

\section{INTRODUCTION}

Rather than the cracked tooth, there is nothing that makes clinicians fall into difficulty from the diagnosis stage. It is hard to grasp the whole picture from outside like a part of an iceberg that has sprung up over the surface of the water. Crack has a different effect on the tooth depending on its degree [1].

Cracked tooth is not easy to diagnose because of the wide variety of symptoms. Crack lines may be difficult to locate; dye staining, transillumination, or microscopy may be necessary to identify crack lines [2,3]. Even if a crack is identified, determining the severity of a crack is an inaccurate prediction rather than an accurate diagnosis and predicting the prognosis of a cracked tooth based on clinical examination is also inaccurate [4]. Planning appropriate treatment for teeth with cracks of unknown depth is a complicated task. Clinicians cannot be sure of the existence of a deep crack until they have actually removed the tooth substance. Therefore, when studying deep cracks, it is most obvious way to observe the inside of the tooth by removal of tooth structure. The pathogenesis of cracked

Received February 1, 2019; Revised March 6, 2019; Accepted March 15, 2019

Corresponding author: Min-Seock Seo, Department of Conservative Dentistry, Wonkwang University Daejeon Dental Hospital, 77 Dunsan-ro, Seo-gu, Daejeon 35233, Korea.

Tel: +82-42-366-1142, Fax: +82-42-366-1115, E-mail: profee@wku.ac.kr

Copyright @ 2019, Oral Biology Research Institute 
tooth was complex and multifactorial for those teeth with or without restorations, with two primary factors predisposing teeth to cracks: natural predisposing features (steep cuspal anatomy, bruxism, clenching, extensive attrition, and abrasion) and iatrogenic features (use of rotary instruments, cavity preparation, and the width and depth of the cavity) [5,6]. As iatrogenic features, restorative procedures may lead to altered strength, fatigue fracture, and cracked tooth by removal of tooth substance. Restorations put teeth at a 29 times greater risk for cracks [7]. However, previous studies have not been clear on this aspect because they did not distinguish between the two factors $[2,8-10]$. Therefore, there is a need to investigate cracks by eliminating iatrogenic factors.

In this study, cases of molar teeth where endodontic treatment was done due to cracked tooth were collected. And then, cases were excluded if the molar had fractures, caries, or fillings. The purpose of this study is to investigate the characteristics of a deep crack in intact teeth without any restoration.

\section{MATERIALS AND METHODS}

This study was approved by the Institutional Review Board of Wonkwang University Daejeon Dental Hospital (W1807/004-001). The molars which were treated endodontically in the Department of Conservative Dentistry at Wonkwang University Daejeon Dental Hospital were screened and evaluated from January 2017 to June 2018.

The cracked teeth were first checked whether there were bite pain and then diagnosed by a bite test using a tooth slooth and wood stick. Examination by naked eye, transillumination, staining with methylene blue dye, and microscopy was also performed to diagnose the crack. The cracked teeth were treated based on pulpal and periapical diagnoses [8]. If the teeth were diagnosed as reversible pulpitis, provisional crowns were placed. If the symptoms resolved, permanent crowns were placed. If the symptoms did not improve after the placement of a provisional crown, endodontic treatment was performed. For cracked teeth diagnosed with irreversible pulpitis or pulp necrosis, endodontic treatment was performed. The teeth diagnosed with vertical root fracture or split teeth were extracted.
Diagnosis and endodontic treatment were performed by 4 endodontists at the Department of Conservative Dentistry at Wonkwang University Daejeon Dental Hospital.

Clinical records of endodontically treated molars were reviewed and the molars that were diagnosed as 'cracked tooth' were selected. From clinical records, only the cases where the crack line was confirmed to extending to the wall of access cavity, were included in the study. And then, teeth with dental caries, restorations, or fractures at the time of diagnosis were excluded. Finally, only cases with reference to a crack line extending to the access cavity wall on clinical records were collected. A retrospective study of 85 cracked molars with intact tooth structure was conducted. Eighty-five clinical records were evaluated in detail (Fig. 1).

General and pretreatment data of these cracked teeth were collected from the clinical records. Age, sex, the location of the tooth, presence of periapical lesion and spontaneous pain, and the history of specific severe pain on chewing and temporomandibular disorder (TMD) were noted. The condition of the molar occluding with the cracked tooth was recorded. In addition, the conditions of contralateral symmetrical molar of the cracked tooth (CSMCT) and the molar which occlude with CSMCT were also recorded. The condition of the tooth was classified as the following 4 types.

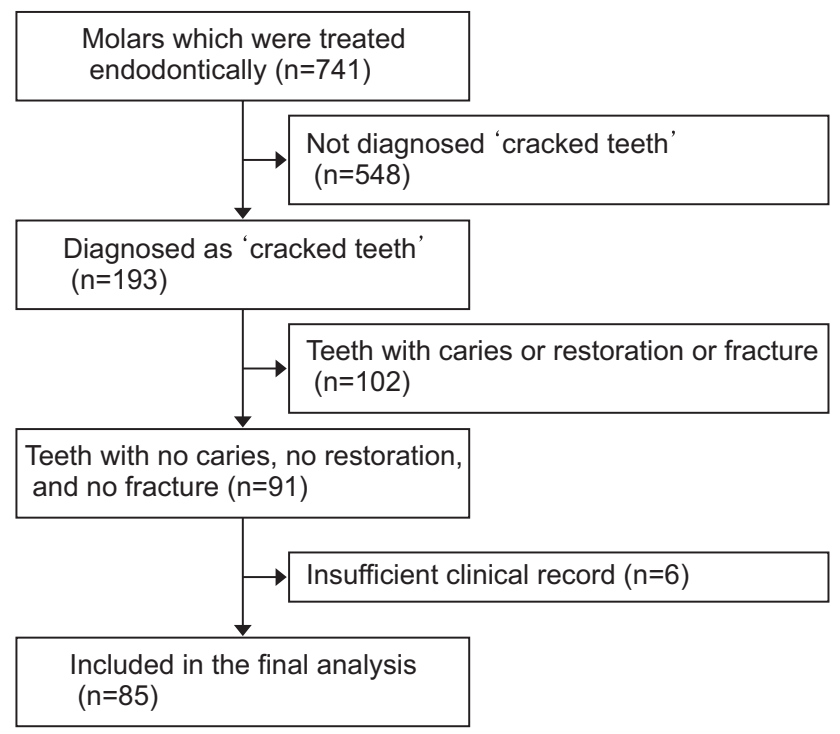

Fig. 1. Stratification of the 741 teeth into 3 mutually exclusive subsets. 
- 'Relatively sound': Molars which have no fracture and restoration or have class 1,2 restoration.

- 'Crack or fracture': Molars which have crack or fracture.

- 'Heavily treated': Molars which have been treated endodontically or have full coverage restoration.

- 'Extracted': Molars which have been extracted.

\section{Statistical analysis}

The obtained results were analyzed using PASW Statistics program ver. 18.0 (IBM Corp., Armonk, NY, USA) and the R statistical language (R Foundation for Statistical Computing, Vienna, Austria) [11]. The chi-square test and independent t-test were used to check the association between variables and spontaneous pain, and to determine the difference in the condition of the occluding molar of cracked tooth, CSMCT, and the occluding molar of CSMCT. In the case that the chi-square test was not applicable, the Fisher exact test was used. The Fisher exact test was used to determine the difference in crack direction according to position (maxilla/mandible). A $p$-value $<0.05$ was considered to be statistically significant.

\section{RESULTS}

Of the 741 teeth examined, 85 teeth were included in the analysis. Fig. 1 depicts the stratification of the 741 teeth into 3 mutually exclusive subsets. Of the total 85 teeth, 58 teeth $(68.2 \%)$ were maxillary molars, which is more than

Table 1. Distribution of cracked tooth according to the location $(\mathrm{n}=85)$

\begin{tabular}{lccc}
\hline \multicolumn{1}{c}{ Type of tooth } & $\begin{array}{c}\text { Male } \\
(\mathbf{n = 7 1 )}\end{array}$ & $\begin{array}{c}\text { Female } \\
(\mathbf{n = 1 4 )}\end{array}$ & Total \\
\hline Maxilla & 51 & 7 & $58(68.2)$ \\
Maxillary first molar & 22 & 3 & $25(29.4)$ \\
Maxillary second molar & 29 & 4 & $33(38.8)$ \\
Mandible & 20 & 7 & $27(31.8)$ \\
Mandibular first molar & 13 & 5 & $18(21.2)$ \\
Mandibluar second molar & 7 & 2 & $9(10.6)$ \\
\hline
\end{tabular}

Values are presented as number only or number (\%). twice as much as mandibular molars. Maxillary second molars $(38.8 \%)$ were the most frequently involved teeth followed by maxillary first molars (29.4\%), mandibular first molars (21.2\%), and mandibular second molars (10.6\%). Cracked teeth were more prevalent among male (83.5\%) than female (Table 1).

The most frequently involved ages were 51 to 60 and 61 to 70 years. In $69.4 \%$, spontaneous pain was not observed. A total of $8.3 \%$ of the patient could not correctly locate the bite pain site. In $14.1 \%$, patients said that symptoms began after feeling a pain when chewing something hard at a certain point (Table 2). More than half of those said that it happened within a week.

Table 3 shows the direction of the crack line found on the wall of access cavity. There was a significant difference between the crack direction of maxillary molars and that of mandibular molars. In $63.0 \%$ of the mandibular molars, a single crack line was observed on the distal region, while the mesio-distal (M-D) transverse crack line and the buccal-side crack line were observed only $3.7 \%$ and

Table 2. Factors of cracked tooth

\begin{tabular}{lc}
\hline \multicolumn{1}{c}{ Characteristic } & Value \\
\hline Age $(y)$ & \\
$21-30$ & $2(2.4)$ \\
$31-40$ & $7(8.2)$ \\
$41-50$ & $16(18.8)$ \\
$51-60$ & $37(43.5)$ \\
$61-70$ & $21(24.7)$ \\
$71-80$ & $2(2.4)$ \\
Spontaneous pain & \\
Absent & $59(69.4)$ \\
Present & $26(30.6)$ \\
Location of bite pain site & \\
Affected arch & $78(91.8)$ \\
Opposite arch & $5(5.9)$ \\
Cannot locate & $2(2.4)$ \\
Periapical lesion & \\
Present & $71(83.5)$ \\
Absent & $14(16.5)$ \\
TMD history & \\
Present & $3(3.5)$ \\
Absent & $82(96.5)$ \\
History of specific severe pain on chewing & \\
Present & $12(14.1)$ \\
Absent & $73(85.9)$ \\
\hline
\end{tabular}

Values are presented as number (\%). 
$7.4 \%$ of the mandibular molar, respectively. In contrast, in the maxillary molars, the M-D transverse crack line was observed in $24.1 \%$ and the palatal-side crack line was observed in $27.5 \%$.

Table 4 shows the relevant factors in relation to the pres-

Table 3. Direction of crack line

\begin{tabular}{lccc}
\hline \multicolumn{1}{c}{ Direction } & $\begin{array}{c}\text { Maxillary } \\
\text { molar } \\
(\mathbf{n = 5 8})\end{array}$ & $\begin{array}{c}\text { Mandibular } \\
\text { molar } \\
(\mathbf{n}=\mathbf{2 7})\end{array}$ & $\boldsymbol{p}^{\text {-value* }}$ \\
\hline Mesial & 3 & 6 & $<0.001$ \\
Distal & 15 & 17 & \\
Mesio-distal transverse & 14 & 1 & \\
Palatal or lingual & 6 & & \\
$\begin{array}{l}\text { Buccal } \\
\text { Combination of mesio-distal } \\
\quad \text { and bucco-lingual direction }\end{array}$ & 10 & 1 & \\
Unknown & 10 & 1 & \\
\hline
\end{tabular}

${ }^{*}$ Fisher's exact test. ence of spontaneous pain. There was a significant difference only in the condition of occluding molar $(p=0.041)$. Table 5 shows the condition of the occluding molar of the cracked tooth, CSMCT, and the occluding molar of CSMCT. There was a significant difference between the condition of CSMCT and that of occluding molars $(p<0.001)$. The percentage of 'relatively sound' in CSMCT (57.6\%) was lower than that in occluding molars $(81.2 \%$ and $76.5 \%)$, while the percentage of 'crack or fracture' in CSMCT (10.6\%) was higher than in occluding molars (0\% and 2.4\%).

\section{DISCUSSION}

There are broadly 3 stages in the life of a crack. These are crack initiation, crack propagation, and fracture. Clinically, it is important to diagnose the presence of a crack line at early stage so that appropriate treatment can be taken to prevent its progression. A variety of diagnostic aids such as

Table 4. Association of variables with cracked tooth according to the presence of spontaneous pain

\begin{tabular}{|c|c|c|c|}
\hline \multirow{2}{*}{ Factor } & \multicolumn{2}{|c|}{ Spontaneous pain } & \multirow{2}{*}{$p$-value } \\
\hline & Absent $(n=59)$ & Present $(n=26)$ & \\
\hline Age & $54.81 \pm 10.77$ & $53.54 \pm 8.57$ & $0.595^{*}$ \\
\hline Sex & & & $0.649^{\dagger}$ \\
\hline Male & $50(70.4)$ & $21(29.6)$ & \\
\hline Female & $9(64.3)$ & $5(35.7)$ & \\
\hline Position & & & $0.138^{\dagger}$ \\
\hline First molar & $33(76.7)$ & $10(23.3)$ & \\
\hline Second molar & $26(61.9)$ & $16(38.1)$ & \\
\hline Location of bite pain site & & & $>.0 .999^{\ddagger}$ \\
\hline Affected arch & $54(69.2)$ & $24(30.8)$ & \\
\hline Wrong arch or cannot locate & $5(71.4)$ & $2(28.6)$ & \\
\hline Mesio-distal transverse crack line & & & $0.134^{\ddagger}$ \\
\hline Absent & $46(65.7)$ & $24(34.3)$ & \\
\hline Present & $13(86.7)$ & $2(13.3)$ & \\
\hline Periapical lesion & & & $0.085^{\dagger}$ \\
\hline Absent & $52(73.2)$ & $19(26.8)$ & \\
\hline Present & $7(50.0)$ & $7(50.0)$ & \\
\hline History of specific severe pain on chewing & & & $0.369^{\dagger}$ \\
\hline Absent & $52(71.2)$ & $21(28.8)$ & \\
\hline Present & $7(58.3)$ & $5(41.7)$ & \\
\hline The condition of occluding molar & & & $0.041^{\ddagger}$ \\
\hline Relatively sound & $39(75.0)$ & $13(25.0)$ & \\
\hline Crack or fracture & $6(100.0)$ & $0(0.0)$ & \\
\hline Heavily treated & $9(60.0)$ & $6(40.0)$ & \\
\hline Extracted & $5(41.7)$ & $7(58.3)$ & \\
\hline
\end{tabular}

Values are presented as mean \pm standard deviation or number (\%).

${ }^{*}$ Independent $\mathrm{t}$-test, ${ }^{\dagger} \chi^{2}$-test, ${ }^{\ddagger}$ Fisher's exact test. 
Table 5. The condition of related tooth with cracked tooth $(n=85)$

\begin{tabular}{lccc}
\hline Condition of tooth & Occluding molar of cracked tooth & CSMCT & Occluding molar of CSMCT $^{p}$-value \\
\hline Relatively sound & $69(81.2)$ & $49(57.6)$ & $65(76.5)$ \\
No restoration & $61(88.4)$ & $39(79.6)$ & $56(86.2)$ \\
Class 1 or 2 restoration & $8(9.4)$ & $10(20.4)$ & $9(13.8)$ \\
Crack or fracture & $0(0.0)$ & $9(10.6)$ & $2(2.4)$ \\
Heavily treated & $12(14.1)$ & $16(18.8)$ & $14(16.5)$ \\
Extracted & $4(4.7)$ & $11(12.9)$ & $4(4.7)$ \\
\hline
\end{tabular}

Values are presented as number (\%) or number only.

CSMCT, contralateral symmetrical molar of cracked tooth. ${ }^{*} \chi^{2}$-test.

staining with methylene blue, transillumination, selective loading of cusps with instruments, and examination under microscopy are useful for the identification of cracks in teeth [6]. Symptoms are also usually definitive. However, there is no consensus on what is a "clinically significant" crack.

From tooth surface, it is unclear how critical a crack line actually is. In this study, the molar on which the patient had bite pain and the crack line was found, was diagnosed as cracked tooth. After endodontic treatment which was done due to persisting pain, only the case of detecting a crack line on the access cavity wall was selected. Therefore, the cracked tooth of this study can be regarded as having a relatively deep crack as compared with the previous studies [2,8-10].

It is controversial whether cracked teeth are more common in maxillary or mandibular molars. Hiatt [12] and Cameron [13] reported that the teeth with the most cracks were mandibular molars. Kim et al. [8] reported that the incidence of cracked tooth was similar in mandibular molars and maxillary molars, and Kang et al. [10] reported that cracked teeth were slightly more common in the mandibular molar. However, Roh and Lee [9] reported that cracked teeth were more common in the maxillary molar. They suggested that because the mandibular molars of Koreans were lingually tilted, the occurrence of cracks was relatively low. In the mandibular molar, there were morphological and positional differences between Europeans and East Asians [14-16], so cracked teeth may appear differently. In this study, it was found that the maxillary molars had crack more than twice as much as the mandibular molars, which supported that East Asians have more cracked teeth in the maxillary molars.

Roh and Lee [9] pointed out that the cracks in intact teeth without restoration and those in largely restored teeth should be to be regarded as different ones, even though they were similar in the early stage. Qing et al. [17] investigated cracked teeth which had no restoration in Chinese population. They reported that the maxillary molars (57.2\%) were more than the mandibular molars (36.3\%), which is similar to the results of this study.

The results of previous studies [2,8-10] differed from the results of this study because they did not confirm how deep the crack actually was. As mentioned earlier, the crack of this study is supposed to be deep cracks, which directly affect the pulp space. Deep cracks of this study were shown to occur more in the maxillary molars. Qian et al. [18] explored the correlation between cuspal inclination and cracked tooth by measuring and reconstructing the cuspal inclinations of cracked maxillary molars through threedimensional finite element analysis. They reported that maxillary molars with steep cuspal anatomy result in an increase in unfavorable stress, therefore, their occurrence of the cracked tooth would be magnified. But the research on this topic is very scarce and more research is needed.

Previous studies $[2,9,12,19]$ have reported that patients are more likely to have cracked teeth at 30 to 50 years of age. In this study, cracked tooth was most common in 51 to 70 years of age. Because this study examined relatively deep cracks, cracked teeth of this study were more prevalent in older patients than in previous studies. The dentin of humans decreased fatigue resistance with age [20]. High prevalence of cracked tooth in older patients appears to be associated with decreased dentin elasticity and increased 
stress fatigue with age $[2,19]$.

A few studies $[2,8,9]$ in East Asian populations have reported no difference in the incidence of cracked tooth in male and female. But, Kang et al. [10] reported that the incidence was higher in male (61\%) than female (39\%). In this study, the incidence rate was 5 times higher in male. It was a common finding in all studies that male show more tooth wear than female. For example, Wetselaar et al. [21] reported that the mean tooth wear scores were 2.00 for male and 1.81 for female and a similar difference was found in all age groups. There is no evidence that the tooth structure differs between male and female, or that there are any differences in the composition of saliva. It might be hypothesized that male's masticatory muscles exert higher forces [22], leading to more mechanical tooth wear and crack. Recently, Lin et al. [23] found consistently higher masticatory muscle thickness in male compared with female. These characteristics seem to be more critical in the case of deep cracks. However, it is not possible to simply explain why wear and crack are more likely to occur in male, and more research about this topic is needed.

Cracks in the occlusal plane occur mainly in the M-D direction. Krishnan et al. [24] reported that coronally, the crack was centered mesio-distally, splitting the pulp chamber into equal halves. In this study, most cracks occurred in the $\mathrm{M}-\mathrm{D}$ direction in mandibular molars. In the maxillary molars, palatal-side crack line was found in $27.5 \%$, which was higher than that in the mandible. The M-D transverse crack line was a very severe pattern of crack and was more pronounced in the maxillary molars. These two features of crack line seem to be related to why the cracked tooth appears more in the maxillary arch.

In this study, we investigated not only the cracked tooth itself, but also CSMCT and the occluding molars of cracked tooth and CSMCT. CSMCT showed more 'crack or fracture' and 'extracted' than two occluding molars. This confirms that teeth that are likely to have natural predisposing features similar to cracked teeth also have a high probability of crack or fracture. Therefore, beyond treating only cracked teeth, clinicians need to actively evaluate and intervene in contralateral symmetrical teeth because it has the same natural predisposing features with cracked tooth. If it is necessary, clinicians are ready to use occlusal adjustment to reduce the stress by reducing cuspal inclination and height. Ratcliff et al. [25] suggest that equilibrating the occlusion and removing interferences wherever appropriate during excursive movements may effectively remove etiologic factors that contribute to the propagation of cracks. Braly and Maxwell [26] recommended reducing cuspal heights to remove traumatic occlusal contacts and remove eccentric contacts. However, to what extent occlusal adjustment should be implemented is still in question.

In this study, iatrogenic cause was excluded, so we could concentrate on the effect of natural predisposing factor. There are factors such as steep cuspal anatomy, bruxism, clenching, extensive attrition, and abrasion in the natural predisposing factor. Qiao et al. [27] reported that the habit of eating hot and cold food alternatively was strongly associated with cracked teeth, whereas eating coarse foods, chewing on hard objects, and unilateral mastication were also independent risk factors for cracked teeth. However, since this study was a retrospective study, the individual data of patients were limited. In this study, the history of having been treated with TMD was identified only in 3.5\% of patients. This figure would have been higher if the patient was specifically interviewed. These factors should be studied through a prospective study in the future.

Deep cracks in intact molar have different characteristics from general cracks. The deep cracks from intact molars occurred two times more in the maxillary molars than in the mandibular molars, and five times more in male than in female. Crack lines observed from access cavity wall were different between maxillary and mandibular molars.

The CSMCTs had 'crack or fracture' or was 'extracted' at a higher rate than the occluding teeth. Therefore, if a cracked tooth is found, it may be important to check the condition of the contralateral tooth and consider the intervention.

\section{ACKNOWLEDGEMENTS}

This research was supported by Wonkwang University in 2019. 


\section{CONFLICTS OF INTEREST}

The authors declare that they have no competing interests.

\section{ORCID}

\author{
Soo-Jeong Hwang \\ https://orcid.org/0000-0003-4725-1512 \\ Min-Seock Seo \\ https://orcid.org/0000-0001-7203-7775
}

\section{REFERENCES}

1. Ricucci D, Siqueira JF Jr, Loghin S, Berman LH. The cracked tooth: histopathologic and histobacteriologic aspects. J Endod 2015;41:343-352. doi: 10.1016/j.joen.2014.09.021.

2. Seo DG, Yi YA, Shin SJ, Park JW. Analysis of factors associated with cracked teeth. J Endod 2012;38:288-292. doi: 10.1016/j.joen.2011.11.017.

3. Özer SY. Detection of vertical root fractures by using cone beam computed tomography with variable voxel sizes in an in vitro model. J Endod 2011;37:75-79. doi: 10.1016/ j.joen.2010.04.021.

4. Berman LH, Kuttler S. Fracture necrosis: diagnosis, prognosis assessment, and treatment recommendations. J Endod 2010;36:442-446. doi: 10.1016/j.joen.2009.12.018.

5. Lynch CD, McConnell RJ. The cracked tooth syndrome. J Can Dent Assoc 2002;68:470-475.

6. Lubisich EB, Hilton TJ, Ferracane J; Northwest Precedent. Cracked teeth: a review of the literature. J Esthet Restor Dent 2010;22:158-167. doi: 10.1111/j.17088240.2010.00330.x.

7. Ratcliff S, Becker IM, Quinn L. Type and incidence of cracks in posterior teeth. J Prosthet Dent 2001;86:168172.

8. Kim SY, Kim SH, Cho SB, Lee GO, Yang SE. Different treatment protocols for different pulpal and periapical diagnoses of 72 cracked teeth. J Endod 2013;39:449-452. doi: 10.1016/j.joen.2012.11.052.

9. Roh BD, Lee YE. Analysis of 154 cases of teeth with cracks. Dent Traumatol 2006;22:118-123. doi: 10.1111/j.16009657.2006.00347.x.

10. Kang SH, Kim BS, Kim Y. Cracked teeth: distribution, characteristics, and survival after root canal treatment. J Endod 2016;42:557-562. doi: 10.1016/j.joen.2016.01.014.

11. R Development Core Team. R: a language and environment for statistical computing. Vienna: R Foundations for Statistical Computing; 2010.

12. Hiatt WH. Incomplete crown-root fracture in pulpalperiodontal disease. J Periodontol 1973;44:369-379. doi: 10.1902/jop.1973.44.6.369.

13. Cameron CE. The cracked tooth syndrome: additional findings. J Am Dent Assoc 1976;93:971-975. doi: 10.14219/ jada.archive.1976.0034.

14. Oh SC. A study on morphology and size of clinical crown of permanent mandibular molar in Korean adult. J Korean Acad Prosthodont 1999;37:242-255.

15. Kook YA, Nojima K, Moon HB, McLaughlin RP, Sinclair PM. Comparison of arch forms between Korean and North American white populations. Am J Orthod Dentofacial Orthop 2004;126:680-686. doi: 10.1016/j.ajodo.2003.10.038.

16. Zhang R, Wang H, Tian YY, Yu X, Hu T, Dummer PM. Use of cone-beam computed tomography to evaluate root and canal morphology of mandibular molars in Chinese individuals. Int Endod J 2011;44:990-999. doi: 10.1111/j.13652591.2011.01904.x.

17. Qing JN, Lu YM, Cao DJ. [Clinical analysis on 274 cases of longitudinal fracture of posterior teeth]. Shanghai Kou Qiang Yi Xue 2003;12:307-309. Chinese.

18. Qian Y, Zhou X, Yang J. Correlation between cuspal inclination and tooth cracked syndrome: a three-dimensional reconstruction measurement and finite element analysis. Dent Traumatol 2013;29:226-233. doi: 10.1111/j.16009657.2012.01160.x.

19. Udoye CI, Jafarzadeh H. Cracked tooth syndrome: characteristics and distribution among adults in a Nigerian teaching hospital. J Endod 2009;35:334-336. doi: 10.1016/ j.joen.2008.12.011.

20. Bajaj D, Sundaram N, Nazari A, Arola D. Age, dehydration and fatigue crack growth in dentin. Biomaterials 2006;27:2507-2517. doi: 10.1016/j.biomaterials. 2005.11.035.

21. Wetselaar P, Vermaire JH, Visscher CM, Lobbezoo F, Schuller AA. The prevalence of tooth wear in the Dutch adult population. Caries Res 2016;50:543-550. doi: $10.1159 / 000447020$.

22. van der Glas HW, Lobbezoo F, van der Bilt A, Bosman F. Influence of the thickness of soft tissues overlying human masseter and temporalis muscles on the electromyographic maximal voluntary contraction level. Eur J Oral Sci 1996;104:87-95. doi: 10.1111/j.1600-0722.1996.tb00051. $\mathrm{x}$.

23. Lin CS, Wu CY, Wu SY, Chuang KH, Lin HH, Cheng DH, Lo WL. Age- and sex-related differences in masseter size and its role in oral functions. J Am Dent Assoc 2017;148:644653. doi: 10.1016/j.adaj.2017.03.001.

24. Krishnan U, Moule A, Michael S, Swain M. Fractographic analysis of a split tooth presenting radiographically as a horizontal root fracture in an unrestored mandibular second molar. J Endod 2018;44:304-311. doi: 10.1016/ j.joen.2017.10.009.

25. Ratcliff S, Becker IM, Quinn L. Type and incidence of cracks in posterior teeth. J Prosthet Dent 2001;86:168- 
172. doi: $10.1067 / \mathrm{mpr} .2001 .116578$.

26. Braly BV, Maxwell EH. Potential for tooth fracture in restorative dentistry. J Prosthet Dent 1981;45:411-414. doi: 10.1016/0022-3913(81)90102-5.
27. Qiao F, Chen M, Hu X, Niu K, Zhang X, Li Y, Wu Z, Shen Z, Wu L. Cracked teeth and poor oral masticatory habits: a matched case-control study in China. J Endod 2017;43:885-889. doi: 10.1016/j.joen.2017.01.007. 